\title{
Monotone iterative technique for a nonlinear fractional $q$-difference equation of Caputo type
}

\author{
Guotao Wang ${ }^{1}$, Weerawat Sudsutad ${ }^{2}$, Lihong Zhang ${ }^{1}$ and Jessada Tariboon ${ }^{2 *}$ (D)
}

"Correspondence:

jessada.t@sci.kmutnb.ac.th

${ }^{2}$ Nonlinear Dynamic Analysis

Research Center, Department of

Mathematics, Faculty of Applied

Science, King Mongkut's University

of Technology North Bangkok,

Bangkok, 10800, Thailand

Full list of author information is

available at the end of the article

\begin{abstract}
By establishing a comparison theorem and applying the monotone iterative technique combined with the method of lower and upper solutions, we investigate the existence of extremal solutions of the initial value problem for fractional $q$-difference equation involving Caputo derivative. An example is presented to illustrate the main result.
\end{abstract}

Keywords: Caputo q-fractional derivative; comparison theorem; monotone iterative sequences; extremal solutions

\section{Introduction}

The quantum calculus (calculus without limits or $q$-calculus) is not of recent appearance. It appeared as a connection between mathematics and physics. The quantum difference operator has a lot of applications in different mathematical areas, such as number theory, combinatorics, special functions, basic hyper-geometric functions, the calculus of variations, control theory, mechanics, and the theory of relativity. For the basic concepts of $q$-calculus, we refer the reader to [1]. Recently, the topic of quantum calculus has attracted the attention of several researchers and a variety of new results can be found in [2-11] and references cited therein.

The monotone iterative technique, combined with the method of lower and upper solutions, is an interesting and effective technique for proving the existence of solutions for initial and boundary value problems of nonlinear differential equations. The basic idea of this method is that by using the upper and lower solutions as an initial iteration, one can construct the monotone sequences for a corresponding linear equation and that converge monotonically to the extremal solutions of the nonlinear equation. So many authors developed the upper and lower solutions methods to solve fractional differential equations; for examples, see [12-26].

Motivated by the above-mentioned work, we investigate the existence of extremal solutions for the following initial value problem of a nonlinear fractional quantum difference equation:

$$
\left\{\begin{array}{l}
{ }_{a}^{C} D_{q}^{\alpha} u(t)=f(t, u(t)), \quad t \in J, \\
u(a)=u_{0},
\end{array}\right.
$$

(C) 2016 Wang et al. This article is distributed under the terms of the Creative Commons Attribution 4.0 International License (http://creativecommons.org/licenses/by/4.0/), which permits unrestricted use, distribution, and reproduction in any medium, provided you give appropriate credit to the original author(s) and the source, provide a link to the Creative Commons license, and indicate if changes were made. 
where ${ }_{a}^{C} D_{q}^{\alpha}$ denotes the Caputo $q$-fractional derivative of order $\alpha, 0<\alpha \leq 1, J=[a, b]$, the function $f \in C(J \times \mathbb{R}, \mathbb{R})$, and the constant $u_{0} \in \mathbb{R}$.

The aim of this paper is to extend the method of upper and lower solutions coupled with the monotone iterative technique to fractional $q$-difference equations. In order to apply the method, we establish a comparison theorem involving the Caputo $q$-fractional derivative, which plays a crucial role in this paper. To the best of our knowledge, it is the first crack at applying the method to a fractional $q$-difference equations of Caputo type.

The rest of paper is organized as follows: In Section 2, we present some preliminary notations, definitions and lemmas that we need in the sequel. In Section 3, we discuss the main result, while an example is presented to illustrate the main result in Section 4.

\section{Preliminaries}

We give some notations, definitions, and preliminary facts which are used throughout the paper.

To begin with, we give some properties of a $q$-shifting operator ${ }_{a} \Phi_{q}(m)=q m+(1-q) a$ that can be found in [9].

Property 2.1 [9] For any $a, m, n \in \mathbb{R}$, and for all positive integer $k$ the following properties hold:

(i) ${ }_{a} \Phi_{q}^{k}(m)={ }_{a} \Phi_{q}^{k-1}\left({ }_{a} \Phi_{q}(m)\right)$ and ${ }_{a} \Phi_{q}^{k}(m)={ }_{a} \Phi_{q^{k}}(m)$ with ${ }_{a} \Phi_{q}^{0}(m)=m$.

(ii) ${ }_{a}(n-m)_{q}^{(0)}=1,{ }_{a}(n-m)_{q}^{(k)}=\prod_{i=0}^{k-1}\left(n-{ }_{a} \Phi_{q}^{i}(m)\right), k \in \mathbb{N} \cup\{\infty\}$.

(iii) $a(n-m)_{q}^{(\gamma)}=n^{(\gamma)} \prod_{i=0}^{\infty} \frac{1-\frac{a}{n} \Phi_{q}^{i}(m / n)}{1-\frac{a}{n} \Phi_{q}^{\gamma+i}(m / n)}$ for $\gamma \in \mathbb{R}$.

We recall some basic concepts of $q$-calculus [9].

The $q$-derivative of a function $f$ on the interval $[a, b]$ is defined by

$$
\left({ }_{a} D_{q} f\right)(t)=\frac{f(t)-f\left({ }_{a} \Phi_{q}(t)\right)}{(1-q)(t-a)}, \quad t \neq a, \quad\left({ }_{a} D_{q} f\right)(a)=\lim _{t \rightarrow a}\left({ }_{a} D_{q} f\right)(t),
$$

and the $q$-derivative of higher order is given by

$$
\left({ }_{a} D_{q}^{0} f\right)(t)=f(t), \quad\left({ }_{a} D_{q}^{k} f\right)(t)={ }_{a} D_{q}^{k-1}\left({ }_{a} D_{q} f\right)(t), \quad k \in \mathbb{N} .
$$

The $q$-derivatives of a product and ratio of functions $f$ and $g$ on $[a, b]$ are

$$
\begin{aligned}
{ }_{a} D_{q}(f g)(t) & =f(t)_{a} D_{q} g(t)+g\left({ }_{a} \Phi_{q}(t)\right)_{a} D_{q} f(t) \\
& =g(t)_{a} D_{q} f(t)+f\left({ }_{a} \Phi_{q}(t)\right)_{a} D_{q} g(t)
\end{aligned}
$$

and

$$
{ }_{a} D_{q}\left(\frac{f}{g}\right)(t)=\frac{g(t)_{a} D_{q} f(t)-f(t)_{a} D_{q} g(t)}{g(t) g\left({ }_{a} \Phi_{q}(t)\right)}, \quad g(t) g\left({ }_{a} \Phi_{q}(t)\right) \neq 0 .
$$

The $q$-integral of a function $f$ defined on the interval $[a, b]$ is given by

$$
\left({ }_{a} I_{q} f\right)(t)=\int_{a}^{t} f(s)_{a} d_{q} s=(1-q)(t-a) \sum_{i=0}^{\infty} q^{i} f\left({ }_{a} \Phi_{q}^{i}(t)\right), \quad t \in[a, b],
$$


with

$$
\left({ }_{a} I_{q}^{0} f\right)(t)=f(t), \quad\left({ }_{a} I_{q}^{k} f\right)(t)={ }_{a} I_{q}^{k-1}\left({ }_{a} I_{q} f\right)(t), \quad k \in \mathbb{N} .
$$

The fundamental theorem of calculus applies to the operator ${ }_{a} D_{q}$ and $I_{q}$, that is,

$$
\left({ }_{a} D_{q a} I_{q} f\right)(t)=f(t)
$$

and if $f$ is continuous at $t=a$, then

$$
\left({ }_{a} I_{q a} D_{q} f\right)(t)=f(t)-f(a) .
$$

The formula for $q$-integration by parts on the interval $[a, b]$ is

$$
\int_{a}^{b} f(s)\left({ }_{a} D_{q} g(s)\right)_{a} d_{q} s=\left.(f g)(t)\right|_{a} ^{b}-\int_{a}^{b} g\left({ }_{a} \Phi_{q}(s)\right)\left({ }_{a} D_{q} f(s)\right)_{a} d_{q} s .
$$

Let us give the definitions of the Riemann-Liouville fractional $q$-derivative and the $q$ integral on the interval $[a, b]$ and their properties [9].

Definition 2.2 [9] The fractional $q$-derivative of Riemann-Liouville type of order $\alpha \geq 0$ on the interval $[a, b]$ is defined by $\left({ }_{a} D_{q}^{0} f\right)(t)=f(t)$ and

$$
\left({ }_{a} D_{q}^{\alpha} f\right)(t)=\left({ }_{a} D_{q^{a}}^{l} I_{q}^{l-\alpha} f\right)(t), \quad \alpha>0
$$

where $l$ is the smallest integer greater than or equal to $\alpha$.

Definition 2.3 [9] Let $\alpha \geq 0$ and $f$ be a function defined on $[a, b]$. The fractional $q$-integral of Riemann-Liouville type is given by $\left({ }_{a} I_{q}^{0} f\right)(t)=f(t)$ and

$$
\left({ }_{a} I_{q}^{\alpha} f\right)(t)=\frac{1}{\Gamma_{q}(\alpha)} \int_{a}^{t}{ }_{a}\left(t-{ }_{a} \Phi_{q}(s)\right)_{q}^{(\alpha-1)} f(s)_{a} d_{q} s, \quad \alpha>0, t \in[a, b],
$$

where the $q$-gamma function is defined by

$$
\Gamma_{q}(\alpha)=\frac{{ }_{0}(1-q)_{q}^{(\alpha-1)}}{{ }_{0}(1-q)_{q}^{\alpha-1}} .
$$

Obviously, $\Gamma_{q}(\alpha+1)=[\alpha]_{q} \Gamma_{q}(\alpha)$.

From [9], we have the following formulas:

$$
\begin{aligned}
& { }_{a} D_{q}^{\alpha}(s-a)^{\beta}(t)=\frac{\Gamma_{q}(\beta+1)}{\Gamma_{q}(\beta-\alpha+1)}(t-a)^{\beta-\alpha}, \\
& { }_{a} I_{q}^{\alpha}(s-a)^{\beta}(t)=\frac{\Gamma_{q}(\beta+1)}{\Gamma_{q}(\beta+\alpha+1)}(t-a)^{\beta+\alpha} .
\end{aligned}
$$


Lemma 2.4 [9] Let $\alpha, \beta \in \mathbb{R}^{+}$and $f$ be a continuous function on $[a, b]$. The RiemannLiouville fractional q-integral has the following semi-group property:

$$
{ }_{a} I_{q}^{\beta}{ }_{a} I_{q}^{\alpha} f(t)={ }_{a} I_{q}^{\alpha} I_{q}^{\beta} f(t)={ }_{a} I_{q}^{\alpha+\beta} f(t)
$$

Lemma 2.5 [9] Let $f$ be a q-integrable function on $[a, b]$. Then the following equality holds:

$$
{ }_{a} D_{q a}^{\alpha} I_{q}^{\alpha} f(t)=f(t), \quad \alpha>0, t \in[a, b] .
$$

Lemma 2.6 [9] Let $\alpha>0$ and $p$ be a positive integer. Then for $t \in[a, b]$ the following equality holds:

$$
{ }_{a} I_{q a}^{\alpha} D_{a}^{p} f(t)={ }_{a} D_{a}^{p} I_{q}^{\alpha} f(t)-\sum_{k=0}^{p-1} \frac{(t-a)^{\alpha-p+k}}{\Gamma_{q}(\alpha+k-p+1)}{ }_{a} D_{q}^{k} f(a) .
$$

Next, the definition of Caputo fractional $q$-derivative is as follows.

Definition 2.7 $[10,11]$ The fractional $q$-derivative of Caputo type of order $\alpha \geq 0$ on the interval $[a, b]$ is defined by $\left({ }_{a}^{C} D_{q}^{0} f\right)(t)=f(t)$ and

$$
\left({ }_{a}^{C} D_{q}^{\alpha} f\right)(t)=\left({ }_{a} I_{q}^{n-\alpha}{ }_{a} D_{q}^{n} f\right)(t), \quad \alpha>0,
$$

where $n$ is the smallest integer greater than or equal to $\alpha$.

Lemma 2.8 $[10,11]$ Let $\alpha>0$ and $n$ be the smallest integer greater than or equal to $\alpha$. Then for $t \in[a, b]$ the following equality holds:

$$
{ }_{a} I_{q a}^{\alpha C} D_{a}^{\alpha} f(t)=f(t)-\sum_{k=0}^{n-1} \frac{(t-a)^{k}}{\Gamma_{q}(k+1)}{ }_{a}^{k} D_{q}^{k} f(a) .
$$

The relation between the Caputo fractional $q$-derivative and the Riemann-Liouville fractional $q$-derivative is given by

$$
{ }_{a}^{C} D_{q}^{\alpha} f(t)={ }_{a} D_{q}^{\alpha}\left[f(t)-\sum_{k=0}^{n-1} \frac{{ }_{a} D_{q}^{k} f(a)}{\Gamma_{q}(k+1)}(t-a)^{k}\right] .
$$

Let $C(J, \mathbb{R})=\{u: J \rightarrow \mathbb{R}: u(t)$ is continuous $\}$ with the norm $\|u\|_{C}=\sup _{t \in J}|u(t)|$.

The following result will play a very important role in this paper.

Lemma 2.9 If $p \in C(J, \mathbb{R})$ and satisfies the following relations:

$$
\left\{\begin{array}{l}
{ }_{a}^{C} D_{q}^{\alpha} p(t) \geq-\lambda p(t), \quad t \in[a, b] \\
p(a) \geq 0
\end{array}\right.
$$

where $\lambda>-\Gamma_{q}(\alpha+1) /(b-a)^{q}$ is a constant, then $p(t) \geq 0$ for all $t \in J$. 
Proof The proof is similar to the argument of Lemma 2.1 in [15]. Assume that $p(t) \geq 0$, $\forall t \in(a, b]$ is not true. Then, by $p(a) \geq 0$, there exist points $t_{0}, t_{1} \in(a, b]$ such that $p\left(t_{0}\right)=0$, $p\left(t_{1}\right)<0, p(t) \geq 0$ for $t \in\left(a, t_{0}\right]$ and $p(t)<0$ for $t \in\left(t_{0}, t_{1}\right]$. Let $t_{2}$ be the first minimal point of $p(t)$ on $\left[t_{0}, t_{2}\right]$. We shall show that $\lambda>0$ and $\lambda \leq 0$.

First of all, let $\lambda>0$. From (2.12), it follows that, for $t_{0}<t \leq t_{1}$,

$$
{ }_{a}^{C} D_{q}^{\alpha} p(t) \geq 0
$$

Using (2.11), Definition 2.2, and equation (2.4), we can compute

$$
\begin{aligned}
{ }_{a}^{C} D_{q}^{\alpha} p(t) & ={ }_{a} D_{q}^{\alpha}[p(t)-p(a)] \\
& ={ }_{a} D_{q a} I_{q}^{1-\alpha}[p(t)-p(a)] \\
& ={ }_{a} D_{q}\left[I_{a} I_{q}^{1-\alpha} p(t)-p(a)_{a} I_{q}^{1-\alpha}(1)(t)\right] \\
& ={ }_{a} D_{q}\left[{ }_{a} I_{q}^{1-\alpha} p(t)-\frac{p(a)(t-a)^{1-\alpha}}{\Gamma_{q}(2-\alpha)}\right] \\
& ={ }_{a} D_{q} H(t),
\end{aligned}
$$

where

$$
H(t)={ }_{a} I_{q}^{1-\alpha} p(t)-\frac{p(a)(t-a)^{1-\alpha}}{\Gamma_{q}(2-\alpha)} .
$$

The fundamental theorem of $q$-calculus applies to the operator $t_{0} I_{q}$, and $t_{0} D_{q}$, we have

$$
{ }_{t_{0}} I_{q t_{0}}^{C} D_{q}^{\alpha} p(t)={ }_{t_{0}} I_{q^{t_{0}}} D_{q} H(t)=H(t)-H\left(t_{0}\right) \geq 0 .
$$

Consequently, for $t \in\left(t_{0}, t_{1}\right]$, it follows that

$$
\begin{aligned}
H(t)-H\left(t_{0}\right)= & {\left[{ }_{a} I_{q}^{1-\alpha} p(t)-\frac{p(a)(t-a)^{1-\alpha}}{\Gamma_{q}(2-\alpha)}\right]-\left[{ }_{a} I_{q}^{1-\alpha} p\left(t_{0}\right)-\frac{p(a)\left(t_{0}-a\right)^{1-\alpha}}{\Gamma_{q}(2-\alpha)}\right] } \\
= & {\left[\frac{1}{\Gamma_{q}(1-\alpha)} \int_{a}^{t}{ }_{a}\left(t-{ }_{a} \Phi_{q}(s)\right)_{q}^{(-\alpha)} p(s)_{a} d_{q} s-\frac{p(a)(t-a)^{1-\alpha}}{\Gamma_{q}(2-\alpha)}\right] } \\
& -\left[\frac{1}{\Gamma_{q}(1-\alpha)} \int_{a}^{t_{0}}{ }_{a}\left(t_{0}-{ }_{a} \Phi_{q}(s)\right)_{q}^{(-\alpha)} p(s)_{a} d_{q} s-\frac{p(a)\left(t_{0}-a\right)^{1-\alpha}}{\Gamma_{q}(2-\alpha)}\right] \\
= & \frac{1}{\Gamma_{q}(1-\alpha)} \int_{a}^{t} a\left(t-{ }_{a} \Phi_{q}(s)\right)_{q}^{(-\alpha)} p(s)_{a} d_{q} s \\
& -\frac{1}{\Gamma_{q}(1-\alpha)} \int_{a}^{t_{0}}{ }_{a}\left(t_{0}-{ }_{a} \Phi_{q}(s)\right)_{q}^{(-\alpha)} p(s)_{a} d_{q} s \\
& -\frac{p(a)}{\Gamma_{q}(2-\alpha)}\left[(t-a)^{1-\alpha}-\left(t_{0}-a\right)^{1-\alpha}\right] \\
\leq & \frac{1}{\Gamma_{q}(1-\alpha)} \int_{a}^{t_{0}}\left(t-{ }_{a} \Phi_{q}(s)\right)_{q}^{(-\alpha)} p(s)_{a} d_{q} s \\
& +\frac{1}{\Gamma_{q}(1-\alpha)} \int_{t_{0}}^{t}{ }_{a}\left(t-{ }_{a} \Phi_{q}(s)\right)_{q}^{(-\alpha)} p(s)_{a} d_{q} s
\end{aligned}
$$




$$
\begin{aligned}
& -\frac{1}{\Gamma_{q}(1-\alpha)} \int_{a}^{t_{0}}{ }_{a}\left(t_{0}-{ }_{a} \Phi_{q}(s)\right)_{q}^{(-\alpha)} p(s)_{a} d_{q} s \\
= & \frac{1}{\Gamma_{q}(1-\alpha)} \int_{a}^{t_{0}}\left[{ }_{a}\left(t-{ }_{a} \Phi_{q}(s)\right)_{q}^{(-\alpha)}-{ }_{a}\left(t_{0}-{ }_{a} \Phi_{q}(s)\right)_{q}^{(-\alpha)}\right] p(s)_{a} d_{q} s \\
& +\frac{1}{\Gamma_{q}(1-\alpha)} \int_{t_{0}}^{t}{ }_{a}\left(t-{ }_{a} \Phi_{q}(s)\right)_{q}^{(-\alpha)} p(s)_{a} d_{q} s .
\end{aligned}
$$

Since $\left[a\left(t-{ }_{a} \Phi_{q}(s)\right)_{q}^{(-\alpha)}-{ }_{a}\left(t_{0}-{ }_{a} \Phi_{q}(s)\right)_{q}^{(-\alpha)}\right]<0$ for $0 \leq s \leq t_{0}$ and $p(t)<0$, for all $t \in\left(t_{0}, t_{1}\right]$, we have

$$
\int_{t_{0}}^{t}{ }_{t_{0}}^{C} D_{q}^{\alpha} p(s)_{t_{0}} d_{q} s<0
$$

which contradicts (2.13). Hence, we obtain the result $\lambda>0$.

Finally, we assume $0 \geq \lambda>-\Gamma_{q}(1+\alpha) /(b-a)^{\alpha}$. Since the Riemann-Liouville fractional $q$-integral ${ }_{a} I_{q}^{\alpha}$ is a monotone operator, thus, applying the fractional $q$-integral order $\alpha$ on both sides of problem (2.12), by using Lemmas 2.8 and 2.4, we have

$$
p(t)-p(a)+\lambda_{a} I_{q}^{\alpha} p(t) \geq 0,
$$

for $t \in(a, b]$, from $p(a) \geq 0$, it follows that

$$
p\left(t_{2}\right)+\lambda_{a} I_{q}^{\alpha} p\left(t_{2}\right) \geq 0
$$

For $t=t_{2}$, we can calculate

$$
\begin{aligned}
{ }_{a} I_{q}^{\alpha} p\left(t_{2}\right)= & \frac{1}{\Gamma_{q}(\alpha)} \int_{a}^{t_{2}}{ }_{a}\left(t_{2}-{ }_{a} \Phi_{q}(s)\right)_{q}^{(\alpha-1)} p(s)_{a} d_{q} s \\
= & \frac{1}{\Gamma_{q}(\alpha)} \int_{a}^{t_{0}}{ }_{a}\left(t_{2}-{ }_{a} \Phi_{q}(s)\right)_{q}^{(\alpha-1)} p(s)_{a} d_{q} s \\
& +\frac{1}{\Gamma_{q}(\alpha)} \int_{t_{0}}^{t_{2}}{ }_{a}\left(t_{2}-{ }_{a} \Phi_{q}(s)\right)_{q}^{(\alpha-1)} p(s)_{a} d_{q} s \\
\geq & \frac{1}{\Gamma_{q}(\alpha)} \int_{t_{0}}^{t_{2}}{ }_{a}\left(t_{2}-{ }_{a} \Phi_{q}(s)\right)_{q}^{(\alpha-1)} p(s)_{a} d_{q} s \\
\geq & \frac{1}{\Gamma_{q}(\alpha)} \int_{t_{0}}^{t_{2}}{ }_{a}\left(t_{2}-{ }_{a} \Phi_{q}(s)\right)_{q}^{(\alpha-1)} p\left(t_{2}\right)_{a} d_{q} s \\
= & \frac{\left(t_{2}-t_{0}\right)^{\alpha}}{\Gamma_{q}(\alpha+1)} p\left(t_{2}\right) \\
\geq & \frac{(b-a)^{\alpha}}{\Gamma_{q}(\alpha+1)} p\left(t_{2}\right),
\end{aligned}
$$

which implies that

$$
\lambda_{a} I_{q}^{\alpha} p\left(t_{2}\right) \leq \frac{\lambda(b-a)^{\alpha}}{\Gamma_{q}(\alpha+1)} p\left(t_{2}\right)
$$


Hence, using (2.14) and (2.15), we obtain

$$
\left(1+\frac{\lambda(b-a)^{\alpha}}{\Gamma_{q}(\alpha+1)}\right) p\left(t_{2}\right) \geq 0
$$

since $1+\lambda(b-a)^{\alpha} / \Gamma_{q}(\alpha+1)>0$, thus, this contradicts the negative property of $p\left(t_{2}\right)$. The proof is completed.

\section{Main results}

Consider the linear initial value problem

$$
\begin{cases}{ }_{a}^{C} D_{q}^{\alpha} u(t)+\lambda u(t)=h(t), & 0<\alpha<1 \\ u(a)=u_{0}, \quad u_{0} \in \mathbb{R}, & t \in J\end{cases}
$$

where $\lambda$ is a given constant and $h \in C(J, \mathbb{R})$.

Lemma 3.1 Let $v_{0}, w_{0}: J \rightarrow \mathbb{R}$ be continuous functions. Assume that $v_{0}, w_{0}$ are lower and upper solutions of (3.1), respectively, and $v_{0} \leq w_{0}$, for all $t \in J$. If

$$
\frac{\lambda(b-a)^{\alpha}}{\Gamma_{q}(\alpha+1)}<1
$$

then the linear initial value problem (3.1) has a unique solution $u \in\left[v_{0}, w_{0}\right]$ on $J$.

Proof The proof consist of two steps.

Step I. We shall show that if $u$ is a solution of (3.1), then $v_{0} \leq u \leq w_{0}$.

Let $p=u-v_{0}$, we get

$$
\left\{\begin{array}{l}
{ }_{a}^{C} D_{q}^{\alpha} p(t) \geq-\lambda p(t) \\
p(a) \geq 0
\end{array}\right.
$$

By Lemma 2.9, $p(t) \geq 0$, for $t \in J$, that is, $u \geq v_{0}$. In the same way, if we set $r=w_{0}-u$, then we can show that $u \leq w_{0}$. Thus, $v_{0} \leq u \leq w_{0}$.

Step II. To prove that problem (3.1) has a unique solution.

Problem (3.1) is equivalent to the following integral equation:

$$
u(t)=u_{0}-\lambda_{a} I_{q}^{\alpha} u(t)+{ }_{a} I_{q}^{\alpha} h(t), \quad \forall t \in J .
$$

Let the operator

$$
A u(t)=u_{0}-\lambda_{a} I_{q}^{\alpha} u(t)+{ }_{a} I_{q}^{\alpha} h(t), \quad \forall t \in J .
$$

For any $u, v \in C(J, \mathbb{R})$, using (2.5), we obtain

$$
\|A u(t)-A v(t)\| \leq \lambda_{a} I_{q}^{\alpha}(1)(b)\|u-v\|_{C}=\frac{\lambda(b-a)^{\alpha}}{\Gamma_{q}(\alpha+1)}\|u-v\|_{C} .
$$


By using (3.2),

$$
\|A u-A v\|_{C}<\|u-v\|_{C} .
$$

Hence the operator $A$ is a contraction in view of the condition (3.2). Consequently, by Banach's fixed point theorem, the operator $A$ has a unique fixed point. That is, problem (3.1) has a unique solution. This completes the proof.

Next, we give the definitions of lower and upper solutions of problem (1.1).

Definition 3.2 A function $v_{0} \in C([a, b], \mathbb{R})$ is called a lower solution of problem (1.1), if it satisfies

$$
\left\{\begin{array}{l}
{ }_{a}^{C} D_{q}^{\alpha} v_{0}(t) \leq f\left(t, v_{0}\right), \quad t \in(a, b], \\
v_{0}(a) \leq u_{0} .
\end{array}\right.
$$

Definition 3.3 A function $w_{0} \in C([a, b], \mathbb{R})$ is called an upper solution of problem (1.1), if it satisfies

$$
\left\{\begin{array}{l}
{ }_{a}^{C} D_{q}^{\alpha} w_{0}(t) \geq f\left(t, w_{0}\right), \quad t \in(a, b], \\
w_{0}(a) \geq u_{0} .
\end{array}\right.
$$

In this paper, we will apply the monotone iterative method to present a result on the existence and uniqueness of the solution of problem (1.1).

Theorem 3.4 Let the function $v_{0}, w_{0} \in C(J, \mathbb{R})$. In addition assume that:

$\left(H_{1}\right) v_{0}$ and $w_{0}$ are lower and upper solutions of problem (1.1), respectively.

$\left(H_{2}\right)$ The function $f \in C(J, \mathbb{R})$ satisfies

$$
f(t, v)-f(t, u) \geq-\lambda(v-u) \quad \text { for } v_{0} \leq u \leq v \leq w_{0},
$$

where $\lambda>\Gamma_{q}(\alpha+1) /(b-a)^{\alpha}$ is a constant.

Then there exist monotone iterative sequences $\left\{v_{n}\right\}$ and $\left\{w_{n}\right\}$, which converge uniformly on the interval $J$ to the extremal solutions of (1.1) in $\left[v_{0}, w_{0}\right]$.

Proof For any $z \in\left[v_{0}, w_{0}\right]$, we consider the following linear IVP problem:

$$
\left\{\begin{array}{l}
{ }_{a}^{C} D_{q}^{\alpha} u(t)=h_{z}(t)-\lambda u(t), \quad t \in(a, b], \\
u(a)=u_{0}
\end{array}\right.
$$

where $h_{z}(t)=f(t, z(t))+\lambda z(t)$ and $\lambda$ is a given constant. Since $v_{0}$ and $w_{0}$ are lower and upper solutions of problem (1.1), by $\left(H_{2}\right)$, we can get

$$
\begin{aligned}
& { }_{a}^{C} D_{q}^{\alpha} v_{0}(t) \leq f\left(t, v_{0}(t)\right) \leq f(t, z(t))+\lambda z(t)-\lambda v_{0}(t)=h_{z}(t)-\lambda v_{0}(t), \\
& v_{0}(a) \leq u_{0}
\end{aligned}
$$


and

$$
\begin{aligned}
& { }_{a}^{C} D_{q}^{\alpha} w_{0}(t) \geq f\left(t, w_{0}(t)\right) \geq f(t, z(t))+\lambda z(t)-\lambda w_{0}(t)=h_{z}(t)-\lambda w_{0}(t), \\
& w_{0}(a) \geq u_{0} .
\end{aligned}
$$

Hence $v_{0}$ and $w_{0}$ are lower and upper solutions of problem (3.1). By Lemma 3.1, we know that problem (3.1) has a unique solution $u \in\left[v_{0}, w_{0}\right]$. Define an operator $A:\left[v_{0}, w_{0}\right] \rightarrow$ $\left[v_{0}, w_{0}\right]$ by $u=A z$.

Next, we shall show that the operator $A$ is nondecreasing. Let $z_{1}, z_{2} \in\left[v_{0}, w_{0}\right]$, such that $z_{1} \leq z_{2}$. Set $\eta=v_{2}-v_{1}, v_{1}=A z_{1}$, and $v_{2}=A z_{2}$. By $\left(H_{2}\right)$, we obtain

$$
\begin{aligned}
{ }_{a}^{C} D_{q}^{\alpha} \eta(t) & ={ }_{a}^{C} D_{q}^{\alpha} \nu_{2}(t)-{ }_{a}^{C} D_{q}^{\alpha} \nu_{1}(t) \\
& =f\left(t, z_{2}(t)\right)+\lambda z_{2}(t)-f\left(t, z_{1}(t)\right)-\lambda z_{1}(t)-\lambda\left(v_{2}(t)-v_{1}(t)\right) \\
& \geq-\lambda\left(z_{2}(t)-z_{1}(t)\right)+\lambda\left(z_{2}(t)-z_{1}(t)\right)-\lambda\left(v_{2}(t)-v_{1}(t)\right) \\
& =-\lambda \eta(t),
\end{aligned}
$$

$\eta(a) \geq 0$.

By Lemma 2.9, $\eta(t) \geq 0$, for $t \in J$. That is, the operator $A$ is nondecreasing.

Now let $v_{n}=A v_{n-1}$ and $w_{n}=A w_{n-1}$ for $n=1,2, \ldots$, then we have

$$
v_{0} \leq v_{1} \leq \cdots \leq v_{n} \leq \cdots \leq w_{n} \leq \cdots \leq w_{1} \leq w_{0} .
$$

It is easy to show that the sequences $\left\{v_{n}(t)\right\}$ and $\left\{w_{n}(t)\right\}$ are uniformly bounded and equicontinuous on $J$. Hence, by Arzela-Ascoli's theorem, we have

$$
\lim _{n \rightarrow \infty} v_{n}(t)=v^{*}(t) \text { and } \quad \lim _{n \rightarrow \infty} w_{n}(t)=w^{*}(t)
$$

uniformly on $t \in J$ and the limit functions $v^{*}, w^{*}$ satisfy problem (1.1). Furthermore, $v^{*}$ and $w^{*}$ satisfy the relation

$$
v_{0} \leq v_{1} \leq \cdots \leq v_{n} \leq v^{*} \leq w^{*} \leq \cdots \leq w_{n} \leq \cdots \leq w_{1} \leq w_{0} .
$$

Finally, we prove that $v^{*}$ and $w^{*}$ are extremal solutions of problem (1.1) in $\left[v_{0}, w_{0}\right]$.

Let $u \in\left[v_{0}, w_{0}\right]$ be any solution of (1.1). Then $A u=u$. Since $v_{0} \leq u \leq w_{0}$ and considering the properties of $A$, i.e., $A$ is nondecreasing, we obtain

$$
v_{n} \leq u \leq w_{n}, \quad n=1,2, \ldots
$$

Taking the limit in (3.7) as $n \rightarrow \infty$, we have $v^{*} \leq u \leq w^{*}$. Therefore $v^{*}, w^{*}$ are the extremal solutions of (1.1) in $\left[v_{0}, w_{0}\right]$. This completes the proof. 


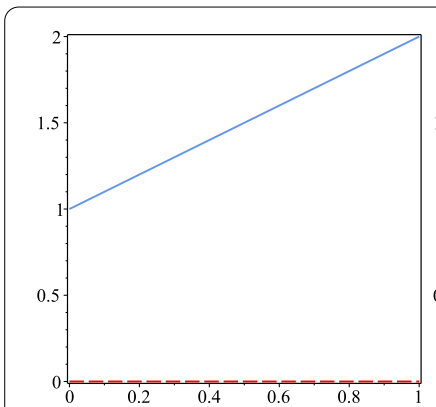

(a) $\left(v_{0}, w_{0}\right)$

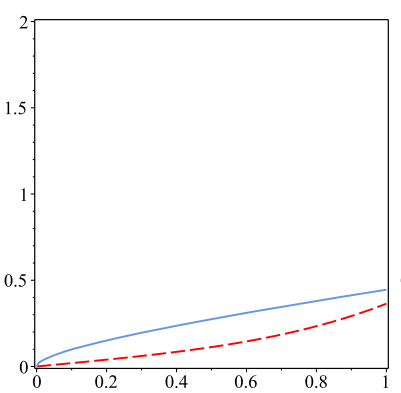

(b) $\left(v_{1}, w_{1}\right)$

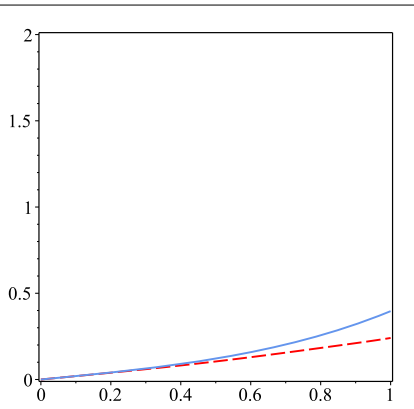

(c) $\left(v_{2}, w_{2}\right)$

Figure 1 A plot of the lower and upper solutions of Problem (4.1).

\section{Examples}

Example 4.1 Consider the following initial value problem:

$$
\left\{\begin{array}{l}
{ }_{0}^{C} D_{q}^{\alpha} u(t)=\frac{t^{1-\alpha}}{3 \Gamma_{q}(1-\alpha)}+\frac{\Gamma_{q}(\alpha+1)}{4}(t-u(t))^{3}, \quad t \in(0,1], \\
u(0)=0
\end{array}\right.
$$

where $\alpha=1 / 2, q=1 / 2, a=0$, and $b=1$. Taking $v_{0}(t)=0$ and $w_{0}(t)=1+t$, it is easy to verify that $v_{0}, w_{0}$ are lower and upper solutions of (4.1), respectively, and $v_{0} \leq w_{0}$. Then the assumption $\left(H_{1}\right)$ of Theorem 3.4 holds.

The function $f$ is given by

$$
f(t, u)=\frac{t^{1-\alpha}}{3 \Gamma_{q}(1-\alpha)}+\frac{\Gamma_{q}(\alpha+1)}{4}(t-u(t))^{3},
$$

which satisfies

$$
f(t, v)-f(t, u) \geq-\frac{3 \Gamma_{q}(\alpha+1)}{4}(v-u)
$$

where $v_{0} \leq u \leq v \leq w_{0}$. Then we get $\lambda=3 \Gamma_{q}(\alpha+1) / 4$, which implies

$$
\frac{\lambda(b-a)^{\alpha}}{\Gamma_{q}(\alpha+1)}=\frac{3}{4}<1
$$

thus, the assumption $\left(H_{2}\right)$ of Theorem 3.4 holds. Therefore, problem (4.1) satisfies all assumptions of Theorem 3.4. By Theorem 3.4, there exist monotone iterative sequences $\left\{v_{n}\right\}$ and $\left\{w_{n}\right\}$, which converge uniformly on interval $[0,1]$ to the extremal solutions of $(4.1)$ in $\left[v_{0}, w_{0}\right]$. The graphs of $\left\{v_{n}\right\}$ and $\left\{w_{n}\right\}$, for some values of $n$, are shown in Figure 1 .

The authors declare that they have no competing interests. 


\section{Acknowledgements}

Article is partially supported by National Natural Science Foundation of China (No. 11501342) and the Scientific and Technological Innovation Programs of Higher Education Institutions in Shanxi (Nos. 2014135 and 2014136).

Received: 22 May 2016 Accepted: 10 August 2016 Published online: 19 August 2016

\section{References}

1. Kac, V: Quantum Calculus. Springer, New York (2002)

2. Annaby, MH, Mansour, ZS: q-Fractional Calculus and Equations. Lecture Notes in Mathematics, vol. 2056. Springer, Berlin (2012)

3. Abdeljawad, T, Baleanu, D: Caputo $q$-fractional initial value problems and a $q$-analogue Mittag-Leffler function. Commun. Nonlinear Sci. Numer. Simul. 16, 4682-4688 (2011)

4. Ferreira, RAC: Nontrivial solutions for fractional $q$-difference boundary value problems. Electron. J. Qual. Theory Differ. Equ. 2010, 70 (2010).

5. Bohner, M, Chieochan, R: Floquet theory for q-difference equations. Sarajevo J. Math. 8(21), 355-366 (2012)

6. Graef, JR, Kong, L: Positive solutions for a class of higher order boundary value problems with fractional $q$-derivatives. Appl. Math. Comput. 281, 9682-9689 (2012)

7. Tariboon, J, Ntouyas, SK: Quantum calculus on finite intervals and applications to impulsive difference equations. Adv. Differ. Equ. 2013, 282 (2013)

8. Ahmad, B, Nieto, JJ, Alsaedi, A, Al-Hutami, H: Existence of solutions for nonlinear fractional q-difference integral equations with two fractional orders and nonlocal four-point boundary conditions. J. Franklin Inst. 351, $2890-2909$ (2014)

9. Tariboon, J, Ntouyas, SK, Agarwal, P: New concepts of fractional quantum calculus and applications to impulsive fractional q-difference equations. Adv. Differ. Equ. 2015, 18 (2015)

10. Ahmad, B, Tariboon, J, Ntouyas, SK, Alsulami, HH, Monaquel, S: Existence results for impulsive fractional quantum difference equations with anti-periodic boundary conditions. Bound. Value Probl. 2016, 16 (2016)

11. Ahmad, B, Ntouyas, SK, Tariboon, J, Alsaedi, A, Alsulami, HH: Impulsive fractional $q$-integro-difference equations with separated boundary conditions. Appl. Math. Comput. 281, 199-213 (2016)

12. Ladde, GS, Lakshmikanthm, V, Vatsala, AS: Monotone Iterative Techniques for Nonlinear Differential Equations. Pitman Pub. Co., Boston (1985)

13. Ahmad, B, Sivasundaram, S: Existence results and monotone iterative technique for impulsive hybrid functional differential systems with anticipation and retardation. Appl. Math. Comput. 197, 515-524 (2008)

14. McRae, FA: Monotone iterative technique and existence results for fractional differential equations. Nonlinear Anal. 71, 6093-6096 (2009)

15. Zhang, S: Monotone iterative method for initial value problem involving Riemann-Liouville fractional derivatives. Nonlinear Anal. 71, 2087-2093 (2009)

16. Wei, Z, Li, Q, Che, J: Initial value problems for fractional differential equations involving Riemann-Liouville sequential fractional derivative. J. Math. Anal. 367, 260-272 (2010)

17. Wang, G: Monotone iterative techniques for boundary value problems of nonlinear fractional differential equation with deviating argument. J. Comput. Appl. Math. 236, 1430-2425 (2012)

18. Wang, G, Baleanu, D, Zhang, L: Monotone iterative method for a class of nonlinear fractional differential equations. Fract. Calc. Appl. Anal. 15, 244-252 (2012)

19. Wang, G, Agarwal, RP, Cabada, A: Existence results and the monotone iterative technique for systems of nonlinear fractional differential equations. Appl. Math. Lett. 25, 1019-1024 (2012)

20. Zhang, L, Ahmad, B, Wang, G: Successive iterations for positive extremal solutions of nonlinear fractional differential equations on a half-line. Bull. Aust. Math. Soc. 91, 116-128 (2015)

21. Nanware, JA, Dhaigude, DB: Monotone technique for finite system of Caputo fractional differential equations with periodic boundary conditions. Dyn. Conti. Dis. Impul. Sys. A 22(1a), 13-23 (2015)

22. Zhang, L, Ahmad, B, Wang, G, Agarwal, RP: Nonlinear fractional integro-differential equations on unbounded domains in a Banach space. J. Comput. Appl. Math. 249, 51-56 (2013)

23. Choudhary, R, Pandey, DN: Monotone iterative technique for neutral fractional differential equation with infinite delay. Math. Methods Appl. Sci. (to appear) doi:10.1002/mma.3901

24. Bai, Z, Zhang, S, Sun, S, Yin, C: Monotone iterative method for fractional differential equations. Electron. J. Qual. Theory Differ. Equ. 6, 8 (2016)

25. Nanware, JA: Existence result for nonlinear initial value problems involving the difference of two monotone functions. Int. J. Anal. Appl. 7(2), 179-184 (2015)

26. Nanware, JA: Existence and uniqueness results for fractional differential equations via monotone method. Bull. Marathwada Math. Soc. 14(1), 39-56 (2013) 